ever users of OCP, and 20.7\% were ever users of HRT. $8.9 \%$ had an aunt on the mother's side with breast cancer, $8.8 \%$ had a sister, and $7.3 \%$ had a mother. $68.2 \%$ were participating for the first time. $88.8 \%$ considered the price acceptable. Television messages and a friend were the most common methods of campaign exposure. Women who participated previously compared to those participating for the first time: were significantly more likely to be older, of higher educational levels, non-smokers, and with a family history of breast cancer.

Conclusion It is essential that governments critically appraise these campaigns in order to enhance outreach, social injustice and equity among the population as well as to ensure better service delivery, capacity and quality.

\section{P1-76 HAI SURVEILLANCE IN SARDINIA, ITALY: POINT- PREVALENCE SURVEY IN A REGIONAL ONCOLOGY CARE CENTRE}

doi:10.1136/jech.2011.142976c.69

\begin{abstract}
${ }^{1} \mathrm{~L}$ Andrissi, ${ }^{*} \mathrm{M}$ P Basciu, ${ }^{1} \mathrm{C}$ Cardia, ${ }^{1} \mathrm{M}$ S Cantini, ${ }^{2} \mathrm{M}$ R Faedda, ${ }^{2} \mathrm{~F}$ Argiolas. ${ }^{1}$ University of Cagliari, Cagliari, Italy; ${ }^{2}$ Health Care Management, "Businco" Hospital, ASL8, Cagliari, Italy
\end{abstract}

Introduction A point-prevalence survey of adult patients was conducted from 14 July to 16 August 2010 in the "BUSINCO" Hospital, a regional primary oncology care centre, to measure the prevalence of Healthcare-Associated Infections (HAIs).

Methods The study consisted of a first phase (30 days), conducted using a daily monitoring system ward by ward and a second phase, starting after hospital discharge and lasting 30 days. International standardised criteria and definitions for the surveillance of HAI were used (CDC).

Results 394 patients were surveyed and the mean length stay was 8.5 days (extra stay of 12.5 if HAI). The most common HAIs were primary bloodstream infections $(32 \%)$, in bone marrow transplantation unit due to coagulase-negative staphylococci), followed by urinary tract $(27 \%)$, respiratory tract $(18 \%)$ and surgical site $(14 \%)$ infections. The use of antibiotics in class I operations (clean), showed that 63 patients $(57 \%)$ received inappropriate prophylactic treatment. A univariate analysis (HAI vs several risk factors : length of stay, urinary catheter, mechanical ventilation, central intravenous catheter) showed a statistically significant association $(p<0.005)$. The multiple logistic regression only showed a significant correlation between HAI and length of stays.

Conclusions Data obtained from this study are representative of an individual setting and our selected activity (immunocompromised patients), necessarily leads to different results than a general hospital. Direct costs of hospitalisation have been proposed as a better method for estimating the cost of hospital-acquired infections and the questionnaire used in this study was added to the hospital discharge registry as a daily routine HAI surveillance tool.

\section{P1-77 MATHEMATICAL AND AGENT-BASED ANALYSES UPON EPIDEMIOLOGICAL DIVERSITY OF THE INCIDENCE OF 2009 NOVEL H1N1 FLU (H1N1) AMONG SCHOOL CHILDREN WITHIN AND AMONG SMALL REGIONAL COMMUNITIES, SAITAMA, JAPAN}

doi:10.1136/jech.2011.142976c.70

R Araki, ${ }^{*}$ M Hanyuu, M Satoh, S Shibazaki, Y Ohno, H Suzuki. Community Health Science Centre, Saitama Medical University, Moroyama, Saitama, Japan

Introduction We examined the epidemiological diversity on the incidence of H1N1 within and among small regional communities using surveillance data and agent-based simulations.
Methods We investigated 27 elementary and junior high schools in Moroyama-town and Sakado-city located in the central part of Saitama Prefecture, Japan. The surveillance system was built on a www server. Agent-based modelling and simulations were performed using AnyLogic 6.5.1 (XJ Technologies, St.Petersburg).

Results By the end of March 2010, cumulative incidence rate (CIR) of $\mathrm{H} 1 \mathrm{~N} 1$ among school children reached $30 \%$ and $34 \%$ in Moroyama and Sakado, respectively. There was no considerable difference between epidemic curves in these neighbouring town and city. On the other hand, in the individual schools, the CIRs ranged 16\%-51\% even if the schools are closely located. To examine the cause of this diversity, we performed agent-based modelling and simulations assuming inequal probability of infection within and outside of schools. Repetitive simulations gave CIRs of $23 \%-44 \%$, indicating that the CIRs of the small population communities may considerably vary even though all the agents were assumed to have the same susceptibility to infection.

Conclusion The granularity of surveillance/analyses/prevention should be finer than in the past to achieve the most effective policies against influenza and similar communicable diseases in the local communities. The cause of this diversity can be explained in part by the stochastic nature of infection transmission processes in the small populations shown by the agent-based simulations. Relevance of the other issues, for example, environmental factors, vaccination, intrafamilial infection, etc, is currently under investigation

\section{P1-78 BODY IMAGE DISSATISFACTION AT EARLY ADOLESCENCE AND CHANGES IN ADIPOSITY THROUGH ADOLESCENCE}

doi:10.1136/jech.2011.142976c.71

\begin{abstract}
${ }^{1,2} \mathrm{~J}$ Araujo, ${ }^{1,2} \mathrm{C}$ Lopes, ${ }^{1,2} \mathrm{E}$ Ramos. ${ }^{*}{ }^{1}$ Department of Hygiene and Epidemiology and Cardiovascular Research \& Development Unit, University of Porto Medical School, Porto, Portugal; ${ }^{2}$ Institute of Public Health, University of Porto, Porto, Portugal
\end{abstract}

Objective To prospectively study the effect of body dissatisfaction on changes in adiposity during adolescence.

Methods We studied 1490 Portuguese adolescents evaluated at 13 and 17 years, under a population-based cohort (EPITeen). Body dissatisfaction was defined as the difference between perceived and desired body image, assessed by Stunkard figures at $13 \mathrm{y}$. BMI z-scores were computed based on CDC percentiles and body fat percentage (BF\%) was assessed using bioelectric impedance. The association between body dissatisfaction and changes in adiposity was computed using linear regression models [regression coefficients $(\beta)$ and $(95 \% \mathrm{CI})]$ and adjusted for adiposity measures at $13 \mathrm{y}$.

Results At age 13 y, 39\% of females desired a thinner image and $16 \%$ desired a larger image. Among males the proportions were $34 \%$ and $33 \%$, respectively. In crude analysis, compared with adolescents who did not have body dissatisfaction, BMI z-scores significantly decreased among adolescents that desired a thinner image $[\beta=-0.152(-0.224 ;-0.080)$ in females and $\beta=-0.206(-0.296$; $-0.117)$ in males]. The opposite association was found among those who desired a larger image $[\beta=0.176(0.081 ; 0.272)$ in females and $\beta=0.113(0.023 ; 0.203)$ in males]. Similar results were found with BF $\%$. However, after adjustment for adiposity measures at $13 \mathrm{y}$, these associations lose significance.

Conclusion We found an association between body image at 13 y and changes in adiposity. The desire of a thinner image was associated with a decrease in adiposity and the desired of a large image associated with an increase. However, the associations were dependent on anthropometric measures at age 13 . 